\title{
Simplified Chinese Version of the Spinal Instability Neoplastic Score in Evaluating Patients with Spinal Metastatic Tumor: A Cross-Cultural Adaptation and Validation
}

\section{Jiajia Chen}

Nantong City No 1 People's Hospital and Second Affiliated Hospital of Nantong University

\section{Mengchen Yin}

Shanghai University of TCM: Shanghai University of Traditional Chinese Medicine

Ningdao Li

Shenzhen Luohu Hospital Group Luohu People's Hospital

\section{Xiangyu Wang}

Nantong City No 1 People's Hospital and Second Affiliated Hospital of Nantong University

\section{Guanhua Xu}

Nantong City No 1 People's Hospital and Second Affiliated Hospital of Nantong University

\section{Guofeng Bao}

Nantong City No 1 People's Hospital and Second Affiliated Hospital of Nantong University

\section{Chongqing $\mathrm{Xu}$}

Shanghai University of TCM: Shanghai University of Traditional Chinese Medicine

Jie Ye

Shanghai University of TCM: Shanghai University of Traditional Chinese Medicine

\section{Junming Ma}

Shanghai University of TCM: Shanghai University of Traditional Chinese Medicine

\section{Wen Mo}

Shanghai University of TCM: Shanghai University of Traditional Chinese Medicine

\section{Dan Wang}

Zhengzhou University First Affiliated Hospital

\section{Zhiming Cui ( $\nabla$ ntspine@ntu.edu.cn )}

Nantong City No 1 People's Hospital and Second Affiliated Hospital of Nantong University https://orcid.org/0000-0002-6032-3640

\section{Research article}


Keywords: Spinal Instability Neoplastic Score, spinal instability, cross-cultural adaptation, reliability, validity

Posted Date: January 18th, 2021

DOl: https://doi.org/10.21203/rs.3.rs-146657/v1

License: (c) (1) This work is licensed under a Creative Commons Attribution 4.0 International License. Read Full License 


\section{Abstract}

Background: The Spinal Instability Neoplastic Score (SINS) in simplified Chinese has not been developed so far. This study aimed to translate the SINS into simplified Chinese, adapt it cross-culturally and to validate its psychometric properties in measuring spinal instability in patients with spinal metastatic tumors in the Chinese mainland.

Methods: The original version of SINS (in English) was translated and cross-culturally adapted into simplified Chinese according to the internationally recognized guidelines. Internal consistency was evaluated with Cronbach's alpha. Test-retest reliability was examined among the patients with a 4-week interval. The validity of the Chinese version of SINS (SC-SINS) was assessed by examining its relationship with Kostuik classification. In addition, floor and ceiling effects were considered present if more than $15 \%$ of respondents achieved the lowest or highest possible total score. Principal component analysis was conducted to confirm the factor structure of each subscale.

Results: No major problems occurred in the forward and back translations of SINS. The internal consistency of SC-SINS was excellent (Cronbach's $a=0.857$, ranging from 0.68 to 0.85 ). Test-retest reliability was also excellent with a value of 0.89 , ranging from 0.86 to 0.95 . Validity analyses indicated that the SC-SINS was positively and significantly correlated with Kostuik classification. All items showed principal component coefficients of over 0.4. No floor or ceiling effects was found in the SC-SINS.

Conclusion: The results indicate that the SC-SINS is reliable and valid in measuring the spinal stability in patients with spinal metastatic tumor.

\section{Background}

For patients with metastatic bone disease, the spine is the most commonly affected site(1,2). Spinal metastasis may lead to spinal cord compression, secondary paralysis and dysfunction, which can bring significantly negative impacts on the patients' quality of life and survival(3). Spinal cord compression from epidural tumor is often considered as an indication for operation. In most spinal metastatic cases, the goal of surgery is not to cure, but to palliatively relieve pain, and to reduce the risk of spinal cord injury.

For spinal tumor surgery, apart from a thorough understanding of the tumor nature and prognosis, evaluating the stability of the lesion segment is also necessary for detailed surgical planning and outcome assessment. In 2010, the Spinal Oncology Study Group (SOSG) defined spinal instability as the "loss of spinal integrity as a result of a neoplastic process that is associated with movement-related pain, symptomatic or progressive deformity, and/or neural compromise under physiological loads" (4). At present, two systems are widely used to assess the spinal instability: the Kostuik classification and Spine Stability Neoplastic Score (SINS). Kostuik classification, which was developed in 1991, divides each vertebra into six columns to determine which lesion may cause mechanical instability and thus require surgical treatment. The six columns include the four columns of the cross section of the vertebral body 
and two columns at the back. It is suggested that spinal instability occurs when the tumor occupies three or more columns, and will be more severe when the tumor involves five or more columns.

The evidence-based Spine Instability Neoplastic Score (SINS) was developed based on the best available literature and expert-opinion consensus(5-7). As an adequate instrument to determine spinal instability, SINS allows easier consultation and communication among specialists treating spinal metastases.

However, SINS, which is in the English language, cannot be accurately understood and accepted by the population in the Chinese mainland. To the best of our knowledge, no reliable and valid simplified Chinese version of the SINS is available at present. Therefore, developing SINS in simplified Chinese can greatly benefit surgical strategy planning and assessment of clinical outcomes for doctors or physicians.

The objective of this study was to translate and cross-culturally adapt the original English version of SINS into simplified Chinese, and to assess the psychometric properties of SC-SINS in patients with metastasis spinal disease. The reliability and validity of SINS in simplified Chinese (SC-SINS) were also examined to demonstrate its accuracy and applicability in clinical practice.

\section{Methods}

\section{Participants}

Patients diagnosed with metastatic spinal disease between January 2016 and January 2020 were recruited. The participant population was advised to be at least 50 for appropriate analysis of reliability, construct validity, as well as ceiling or floor effects, and 100 patients are needed for internal consistency analysis(8). Patients participating in the study were all diagnosed with metastatic spinal disease confirmed by pathology or PET-CT. Eight spine evaluators are at least 6 years of education and ability to read and speak Chinese. Patients excluded were those who reported a history of spinal surgery or whose spinal disease was caused by infection, ankylosing spondylitis, or systemic rheumatologic disease. Complete imaging results and available clinical data were required for all patients. Complete imaging results included sagittal view, axial view and coronal view of magnetic resonance imaging (MRI). Clinical data included demographic characteristics, neurological function, tumor levels, pathology report, complications and surgical treatment. The study was approved by the Ethics Committee of our institution, and all patients signed a written informed consent.

\section{Instruments}

\section{SINS}

SINS, a comprehensive classification to diagnose neoplastic spinal instability, comprises six individual component scores: spine location, pain, lesion bone quality, radiographic alignment, vertebral body collapse, and posterolateral involvement of the spinal elements. The maximum score is 18 , and the minimum is 0 . The total score is divided into three categories in terms of stability: stable (0-6 points), potentially unstable (7-12 points), and unstable (13-18 points). In addition, the SINS score can also be 
analyzed as a binary indicator of surgical referral status: 'stable' (0-6 points) or 'current or possible instability' (7-18 points). A surgical consultation is recommended for patients with SINS scores greater than 7.

\section{Kostuik classification}

The Kostuik classification was used to classify the degree of tumor involvement of the spinal column. The vertebral body is divided into 6 components, and three categories of stability are considered: stable (1-2 partial damages), relatively unsteady (3-4 partial damages) and absolutely unsteady (5-6 partial damages).

\section{Procedure}

The study was conducted in two phases: first, the SINS was translated into simplified Chinese; second, the factor structure, internal consistency, test-retest reliability, validity, and floor and ceiling effects of the SC-SINS were assessed. The procedures followed the cross-cultural adaptation guidelines issued by the American Association of Orthopedic Surgeons Outcome Committee.

\section{Stage I: Forward translation into simplified Chinese}

The 6-component SINS was translated into simplified Chinese independently by 2 bilingual translators who spoke Chinese as the first language. One translator was a medical professional who knew the concepts related to the index well; the other translator was a professional translator with no medical background and was blind for the objective of the study.

\section{Stage II: Synthesis of the translations}

The expert committee, consisting of translators, radiologist and spinal surgeons specialized in metastatic spinal disease discussed the translations and compared them with the original English version SINS. After reaching a consensus, the forward translations were formulated into one single simplified Chinese version.

\section{Stage III: Backward translation into English}

Then backward translation was undertaken independently by another professional bilingual translator, a radiologist and a spinal orthopedist. All lacked medical backgrounds and were not aware of the prior translation procedures. They independently and blindly translated the simplified Chinese version back into English. Each English translation was then compared with the original English version and checked for inconsistencies.

\section{Stage IV: Expert committee}

The expert committee consolidated all the translations and discussed with all the translators, bilingual experts and spinal surgeons. A consensus was reached on all discrepancies. Then the committee came 
into an agreement on the equivalence between the original version and the target version. Finally, the SCSINS was created.

\section{Stage V: Evaluation of the pre-final version}

The data of 28 patients were collected for pilot test by evaluators. Each subsequently pointed out their difficulties in completing the classification or understanding the purpose and meaning of each question. The expert committee discussed all the findings and then developed the final version of SC-SINS which was used for further psychometric testing.

\section{Stage Vl: Evaluation of the final version}

A booklet that included the final SC-SINS and informed consent form was given to all participating patients who met the inclusion/exclusion criteria. We evaluated the internal consistency, test-retest reliability, and floor and ceiling effects of the final version. Each patient's demographic characteristics were recorded.

\section{Statistical analysis}

SPSS18.0 (Chicago, IL, USA) was used for statistical analysis. Data were expressed as the mean \pm standard deviation (SD). Values were reported with $95 \%$ confidence intervals (Cls) and P-values $<0.05$ indicated statistical significance.

\section{Internal consistency}

Internal consistency reliability was evaluated using Cronbach's a coefficient for each domain(9). High Cronbach's $a$ indicated high correlations among the items. Cronbach's $a$ of $\geq 0.70$ was considered satisfactory. In addition, the item-total correlations of each item were calculated. Levels of agreement for $a$ were graded according to the recommendations of Landis and Koch, with a value of 0.00 to 0.20 considered slight agreement; 0.21 to 0.40 , fair agreement; 0.41 to 0.60 , moderate agreement; 0.61 to 0.80 , substantial agreement; and 0.81 to 1.00 , almost perfect agreement(10).

\section{Test-retest reliability}

The test-retest reliability was assessed by comparing the results of the first and final SC-SINS scales. Two-way ANOVA random-effects intra-class correlation coefficient (ICC) was calculated to quantify the test-retest reliability $(11,12)$. We assessed 60 patients for a second time after the first completion. The sequence of SC-SINS was rearranged to reduce the memory error. A 4-week interval was designed between the two tests. ICC values ranged from 0 to 1 , and a higher value indicated higher repeatability. An ICC above 0.7 could be accepted as good and below 0.4 as poor reliability(13). The correlation values were as follows: $0-0.20$, poor; $0.21-0.40$, fair; $0.41-0.60$, moderate; $0.61-0.80$, very good; $0.81-1.0$, excellent.

\section{Validity}


To assess criterion-related validity, we examined construct validity. We evaluated the relationship between the SC-SINS and Kostuik classification using the Pearson correlation coefficients. Correlation values of 0.81-1.0 was considered excellent, $0.61-0.80$ very good, $0.41-0.60$ good, $0.21-0.40$ fair, and $0-0.20$ poor.

\section{Structural factor analysis}

We used factor analysis to evaluate the factor structure of the SC-SINS, and to confirm the subscales. Since the original SC-SINS indicated that the items were distributed across six subscales, we used principal component analysis rotation to confirm the factor structure of each subscale, rather than exploratory factor analysis. Item loadings on each factor equal to or greater than 0.4 were considered satisfactory. In addition, floor and ceiling effects (defined as the percentage of participants displaying the minimum and maximum possible scores, respectively) were calculated and those with over $15 \%$ of respondents achieving the lowest or highest possible total scores were considered significant(11).

\section{Results}

\section{Translation and cross-cultural adaptation}

No major problems occurred in the forward and back translations of SINS. The minor differences caused by cultural differences in some items were then adapted cross-culturally and some modifications were also made. In section 2 (Pain), "occasionally, not consistently. No severe pain, and it was tolerable" was added as the explanation.

In pilot trial, 28 classifications that included suggestions about the pre-final SC-SINS were asked. Among the 28 participants (12 males and 16 females), 17 received surgical treatment and 11 received nonsurgical treatment. Table 1 summarized the patients' characteristics. Among the 28 patients, 13 mistakenly considered that the items were asking about the severity of pain before or after surgery. After consulting the expert committee, we revised the pre-final SC-SINS and emphasized "pain" referred to the preoperative pain for patients undergoing surgical treatment. Finally, the simplified Chinese version of the SINS was produced.

\section{Demographic and clinical characteristics of the sample}

A total of 160 participants (88males and 72 females) were enrolled in the final test. The mean age, gender, duration and BMI of the pre-final, test-retest, and validity groups were similar. The number of participants with Frankel Score (A-C) were 16, 17, 73 in the three group respectively. Pathologically, the primary tumors were mostly lung, breast, liver, renal, gastric, intestinal tumors. The demographic data of the participants in each group and descriptive statistics were shown in Table 1. 
Table 1

Demographic and clinical characteristics of the sample

\begin{tabular}{|c|c|c|c|}
\hline Characteristic Group & $\begin{array}{l}\text { Prefinal group } \\
(\mathrm{n}=28)\end{array}$ & $\begin{array}{l}\text { Test-retest group } \\
(\mathrm{n}=33)\end{array}$ & $\begin{array}{l}\text { Validity group } \\
(n=160)\end{array}$ \\
\hline Age, years & $59.5 \pm 8.8$ & $62.5 \pm 9.5$ & $60.5 \pm 8.2$ \\
\hline Gender, Male/Female & $12 / 16$ & $18 / 15$ & $88 / 72$ \\
\hline Disease duration,months & $12.5 \pm 4.8$ & $15.2 \pm 3.9$ & $13.1 \pm 4.1$ \\
\hline BMI (kg/m2) & $23.7 \pm 1.8$ & $22.5 \pm 2.1$ & $22.8 \pm 2.3$ \\
\hline Occupation, active/retired & $16 / 12$ & $17 / 16$ & $73 / 87$ \\
\hline \multicolumn{4}{|l|}{ Primary tumor } \\
\hline lung/breast & 8 & 10 & 35 \\
\hline liver/renal & 6 & 3 & 29 \\
\hline gastric/intestinal & 6 & 8 & 28 \\
\hline other & 8 & 12 & 68 \\
\hline Tumor stage, I-III/IV & $20 / 8$ & $22 / 11$ & $110 / 50$ \\
\hline Frankel Score, A-C/D-E & $15 / 13$ & $16 / 17$ & $85 / 75$ \\
\hline Segments involved, Single/multiple & $14 / 14$ & $19 / 14$ & $78 / 82$ \\
\hline Visceral metastasis, yes/no & $9 / 21$ & $11 / 22$ & $56 / 104$ \\
\hline Treatment, Surgical/Non-Surgical & $17 / 11$ & $18 / 15$ & $91 / 69$ \\
\hline \multicolumn{4}{|l|}{ SINS Component } \\
\hline \multicolumn{4}{|l|}{ Location } \\
\hline Junctional & 2 & 5 & 28 \\
\hline Mobile spine & 12 & 12 & 64 \\
\hline Semirigid & 12 & 11 & 56 \\
\hline Rigid & 2 & 5 & 12 \\
\hline \multicolumn{4}{|l|}{ Pain } \\
\hline Yes & 20 & 22 & 132 \\
\hline Occasional pain but not mechanical & 7 & 9 & 19 \\
\hline Pain-free lesion & 1 & 2 & 9 \\
\hline
\end{tabular}




\begin{tabular}{|l|lll|}
\hline Lytic & 9 & 14 & 68 \\
\hline Mixed & 11 & 15 & 72 \\
\hline Blastic & 8 & 4 & 20 \\
\hline Radiographic Sal Alignment & 3 & 6 & 13 \\
\hline Subluxation/translation present & 11 & 9 & 27 \\
\hline De novo deformity & 14 & 18 & 120 \\
\hline Normal alignment & & & 68 \\
\hline Vertebral Body Collapse & 11 & 7 & 50 \\
\hline$>50 \%$ collapse & 9 & 12 & 18 \\
\hline$<50 \%$ collapse & 4 & 11 & 9 \\
\hline No collapse with $>50 \%$ body involved & 4 & 3 & 80 \\
\hline None of the above & & & 70 \\
\hline Posterolateral Involvement of Spinal Elements & 15 & 16 & 10 \\
\hline Bilateral & 11 & 2 & \\
\hline Unilateral & 2 & & \\
\hline None of the above & & & \\
\hline
\end{tabular}

\section{Internal consistency}

The internal consistency of SC-SINS was excellent (Cronbach's $a=0.857)$. All the item-total score correlations were moderate to high, ranging from 0.68 (Pain, item 2) to 0.85 (Location, item2). The Cronbach's $a$ with elimination of one item did not increase by more than 0.1 for each item, indicating that all items were relevant to this population. Table 2 demonstrated the results for the Cronbach's $a$, item-total score correlation, and Cronbach's $a$ if the item was deleted.

\section{Test-retest reliability}

The retest session included 33 randomly selected patients and the interval between the two tests was 4 weeks. The scores for the retest were similar with those in the first test. All the ICC values were high, ranging from 0.86 (Radiographic spinal alignment) to 0.95 (Location). The ICC values of total SC-SINS was 0.89 (95\% Cl: 0.87-092), indicating excellent test-retest reliability (Table 2). 
Table 2

The internal consistency and Test-retest reliability of SC-SINS

\begin{tabular}{|c|c|c|c|c|c|}
\hline \multirow[t]{2}{*}{ SC-SINS } & \multirow[t]{2}{*}{ Item } & \multicolumn{2}{|c|}{$\begin{array}{l}\text { Item-total Score } \\
\text { Correlation }(n=160)\end{array}$} & \multirow[t]{2}{*}{$\begin{array}{l}\text { Cronbach's a } F \text { Item } \\
\text { Deleted }(n=160)\end{array}$} & \multirow{2}{*}{$\begin{array}{l}\text { ICC } \\
\text { Values } \\
(n=33)\end{array}$} \\
\hline & & $\mathbf{R}$ & $\mathbf{P}$ & & \\
\hline \multirow[t]{4}{*}{ Location } & Q1 & 0.79 & $<0.0001$ & 0.925 & $\begin{array}{l}0.96 \\
(0.94- \\
0.99)\end{array}$ \\
\hline & Q2 & 0.85 & $<0.0001$ & 0.936 & $\begin{array}{l}0.95 \\
(0.93- \\
0.97)\end{array}$ \\
\hline & Q3 & 0.77 & $<0.0001$ & 0.893 & $\begin{array}{l}0.97 \\
(0.94- \\
0.99)\end{array}$ \\
\hline & Q4 & 0.74 & $<0.0001$ & 0.914 & $\begin{array}{l}0.93 \\
(0.90- \\
0.95)\end{array}$ \\
\hline \multirow[t]{3}{*}{ Pain } & Q1 & 0.71 & $<0.0001$ & 0.818 & $\begin{array}{l}0.87 \\
(0.83- \\
0.92)\end{array}$ \\
\hline & Q2 & 0.68 & $<0.0001$ & 0.826 & $\begin{array}{l}0.93 \\
(0.91- \\
0.94)\end{array}$ \\
\hline & Q3 & 0.79 & $<0.0001$ & 0.825 & $\begin{array}{l}0.90 \\
(0.85- \\
0.95)\end{array}$ \\
\hline \multirow[t]{3}{*}{ Bone lesion } & Q1 & 0.76 & $<0.0001$ & 0.876 & $\begin{array}{l}0.89 \\
(0.85- \\
0.92)\end{array}$ \\
\hline & Q2 & 0.75 & $<0.0001$ & 0.899 & $\begin{array}{l}0.93 \\
(0.90- \\
0.95)\end{array}$ \\
\hline & Q3 & 0.81 & $<0.0001$ & 0.902 & $\begin{array}{l}0.91 \\
(0.88- \\
0.94)\end{array}$ \\
\hline \multirow[t]{3}{*}{$\begin{array}{l}\text { Radiographic spinal } \\
\text { alignment }\end{array}$} & Q1 & 0.71 & $<0.0001$ & 0.893 & $\begin{array}{l}0.88 \\
(0.84- \\
0.92)\end{array}$ \\
\hline & Q2 & 0.73 & $<0.0001$ & 0.816 & $\begin{array}{l}0.84 \\
(0.81- \\
0.90)\end{array}$ \\
\hline & Q3 & 0.81 & $<0.0001$ & 0.825 & $\begin{array}{l}0.89 \\
(0.83- \\
0.92)\end{array}$ \\
\hline
\end{tabular}




\begin{tabular}{|c|c|c|c|c|c|}
\hline \multirow[t]{4}{*}{ Vertebral body collapse } & Q1 & 0.71 & $<0.0001$ & 0.796 & $\begin{array}{l}0.86 \\
(0.81- \\
0.90)\end{array}$ \\
\hline & Q2 & 0.73 & $<0.0001$ & 0.897 & $\begin{array}{l}0.88 \\
(0.83- \\
0.91)\end{array}$ \\
\hline & Q3 & 0.72 & $<0.0001$ & 0.814 & $\begin{array}{l}0.84 \\
(0.81- \\
0.87)\end{array}$ \\
\hline & Q4 & 0.81 & $<0.0001$ & 0.907 & $\begin{array}{l}0.85 \\
(0.81- \\
0.87)\end{array}$ \\
\hline \multirow[t]{3}{*}{$\begin{array}{l}\text { Posterolateral involvement } \\
\text { of spinal elements }\end{array}$} & Q1 & 0.72 & $<0.0001$ & 0.796 & $\begin{array}{l}0.87 \\
(0.83- \\
0.92)\end{array}$ \\
\hline & Q2 & 0.76 & $<0.0001$ & 0.858 & $\begin{array}{l}0.88 \\
(0.86- \\
0.90)\end{array}$ \\
\hline & Q3 & 0.71 & $<0.0001$ & 0.826 & $\begin{array}{l}0.91 \\
(0.89- \\
0.94)\end{array}$ \\
\hline
\end{tabular}

SC-SINS indicates simplified Chinese version of SINS; Cronbach's $=0.857$

\section{Validity}

Validity analyses indicated that the SC-SINS was positively and significantly correlated with Kostuik classification $(P<0.0001)$. The six components of the SC-SINS were also significantly associated with the three components of the Kostuik classification (all $P$ values $<0.0001$ ). Table 3 showed that the correlations between "Posterolateral Involvement of Spinal Elements" and "1-2 Partial Damage" was the highest with a correlation value of 0.792 . The correlations between "Pain" and "1-2 Partial Damage" was the lowest with a value of 0.341 . 
Table 3

Pearson Correlation Coefficient of the SC-SINS with Kostuik Classification

\begin{tabular}{|llll|}
\hline Factor & $\begin{array}{l}\text { 1-2 Partial } \\
\text { Damage }\end{array}$ & $\begin{array}{l}\text { 3-4 Partial } \\
\text { Damage }\end{array}$ & $\begin{array}{l}\text { 5-6 Partial } \\
\text { Damage }\end{array}$ \\
\hline Location & 0.741 & 0.572 & 0.523 \\
\hline Pain & 0.341 & 0.623 & 0.499 \\
\hline Bone lesion & 0.451 & 0.622 & 0.381 \\
\hline Radiographic Spinal Alignment & 0.381 & 0.546 & 0.381 \\
\hline Vertebral Body Collapse & 0.541 & 0.712 & 0.689 \\
\hline $\begin{array}{l}\text { Posterolateral Involvement of Spinal } \\
\text { Elements }\end{array}$ & 0.792 & 0.672 & 0.598 \\
\hline
\end{tabular}

\section{Factor analysis}

Table 4 summarized the results of the factor analysis of all the SC-SINS items. All items showed principal component coefficients of over 0.4 . The values of Factors 1 ranged from 0.523 to 0.681 ; Factors 2 ranged from 0.591 to 0.731 ; Factors 3 ranged from 0.613 to 0.754 ; Factors 4 ranged from 0.461 to 0.711 ; Factors 5 ranged from 0.513 to 0.701 and Factors 6 ranged from 0.501 to 0.668 . In addition, neither floor nor ceiling effects were seen in the SC-SINS. 
Table 4

Factor Analyses for the SC-SINS Items

\begin{tabular}{|lr}
\hline SC-SINS & Factor \\
\hline Factor 1 & Location \\
\hline Q 1-1 & 0.681 \\
\hline Q 1-2 & 0.584 \\
\hline Q 1-3 & 0.523 \\
Q 1-4 & 0.546 \\
\hline
\end{tabular}

\section{Factor 2 Pain}

$\begin{array}{ll}\text { Q 2-1 } & 0.591 \\ \text { Q 2-2 } & 0.676 \\ \text { Q 2-3 } & 0.731\end{array}$

Factor 3 Bone lesion

$\begin{array}{ll}\text { Q 3-1 } & 0.613 \\ \text { Q 3-2 } & 0.681 \\ \text { Q 3-3 } & 0.754\end{array}$

Factor 4 Radiographic Sal Alignment

$\begin{array}{ll}\text { Q 4-1 } & 0.565 \\ \text { Q 4-2 } & 0.461 \\ \text { Q 4-3 } & 0.711\end{array}$

Factor 5 Vertebral Body Collapse

$\begin{array}{ll}\text { Q 5-1 } & 0.513 \\ \text { Q 5-2 } & 0.626 \\ \text { Q 5-3 } & 0.619 \\ \text { Q 5-4 } & 0.701\end{array}$

Factors 6 Posterolateral Involvement of Spinal Elements

$\begin{array}{ll}\text { Q 6-1 } & 0.668\end{array}$

Q 6-2 0.592

$\begin{array}{ll}\text { Q 6-3 } & 0.501\end{array}$ 


\section{Discussion}

The recent years have witnessed a sharp increase in the quantity and quality of clinical research in China. Therefore, valid scoring systems are urgently needed to support the clinical research in China. However, there is no "gold standard" for the use of the scoring system should be used, and the doctors' views on spinal stability vary greatly. SINS is a valuable tool to quantify the patients' spinal stability and conduct data analysis. This study described the process of cross-cultural adaptation, structural validity, reliability and construct validity of SINS in Chinese-speaking subjects. The unidimensional scale displayed satisfactory reliability and construct validity in patients with spinal metastatic tumor.

Following the recommended guidelines, the translation and cross-cultural adaptation was successfully conducted. The comprehensibility of the translated items was then confirmed, thus leading to a valid measure of spinal stability, allowing comparability of the data and cross-national studies. The SC-SINS was easy to understand and simple to use. After minor modifications, no item was hard for participants to understand, and all items were answered in pretest and formal study, which revealed good acceptability of SC-SINS. No significant floor or ceiling effects were found in of SC-SINS.

In our study, Cronbach's a reached 0.857 , indicating a perfect internal consistency. This value is similar to those in other studies on SINS. Xu et a/ reported that the Cronbach's a was 0.831(14). Pennington $Z$ et al reported a value of 0.815 for the reliability of SINS score(15). Fox $S$ et al indicated that the total SINS score was nearly perfect with a Cronbach's a of $0.990(16)$. Arana E et al reported that the agreement was perfect with ICC $=0.96(17)$. The item-total score correlations were moderate to high (ranging from 0.68 to 0.85 ), and when a single item was deleted, the deletion did not increase the Cronbach's a by more than 0.1 . Those findings suggested greater homogeneity of all sections and that each section was well correlated to the SC-SINS. Item 1, a question based on objective fact, had the highest ICC value (Location, ICC $=0.85$ ), which indirectly revealed the success of cross-cultural adaptation. However, item 2 presented the lowest ICC value (Pain, ICC $=0.68$ ). One possible explanation might be that the feeling of pain is subjective, which may change with the different treatment. The test-retest reliability was excellent in this study. The ICC value of our study reached 0.89 , which is higher than that in the abovementioned studies. Item 3, another subjective item, presented the lowest ICC value (Radiographic spinal alignment, ICC = 0.86), which could be attributed to the change caused by different body positions or other situations.

The correlation between the components of SC-SINS and Kostuik classification was in accordance with our hypothesis that SC-SINS was positively and significantly correlated with Kostuik classification. According to the results of our study, the association between "Location" and "Posterolateral Involvement of Spinal Elements" of SC-SINS and "1-2 Partial Damage" of Kostuik Classification was the strongest ( $r$ $=0.741$ and 0.792 , respectively). One possible reason might be that these two components are both designed to evaluating tumor involvement, and both of them are important for developing surgical strategies. 
The limitations of our study must be noted. First, the participants in this study could not represent the entire Chinese population with spinal metastatic tumor. The patients were recruited from only four institutions, so enrollment of participants from multiple institutions would provide better sampling and improve the generalizability. However, the variability of this study was enough to demonstrate responsiveness. Second, the clinical experience of evaluators can affect the accuracy. Different understanding on systems may lead to a deviation in the results. Skilled spinal tumor surgeons should have been invited as evaluators to minimize bias, because they can make an agreement more easily than unskilled surgeons(18).

\section{Conclusions}

The cross-cultural adaption of SINS into simplified Chinese was successful. The SC-SINS demonstrated high internal consistency and test-retest reliability. The SC-SINS has been proven valid and reliable to measure spinal stability of patients with spinal metastatic tumor in the population who uses simplified Chinese.

\section{Abbreviations}

SINS: Spinal Instability Neoplastic Score

\section{Declarations}

\section{Acknowledgements}

The authors wish to thank the National Natural Science Foundation of China (grant numbers: 81702216 and 81571828), the construction of famous traditional Chinese medicine doctor "Mo Wen" and workshop in Pudong New Area (Number: PDZYXK-3-2014018). All authors have approved the final version of the manuscript and read the journal's authorship agreement.

\section{Conflicts of interest}

The authors declare that they have no conflicts of interest.

\section{Author Contributions}

Jiajia Chen, Mengchen Yin and Ningdao Li designed the study. Xiangyu Wang, Guanhua Xu, Guofeng Bao, Chongqing $\mathrm{Xu}$, Junming Ma, Jie Ye, Ningdao Li and Dan Wang collected the data. Wen Mo, Dan Wang and Zhiming Cui did the date analysis. Mengchen Yin and Jiajia Chen wrote the manuscript. Jiajia Chen, Dan Wang and Zhiming Cui revised the manuscript. Wen Mo and Zhiming Cui decided to submit the manuscript for publication.

\section{Funding}


This study was supported by National Natural Science Foundation of China (grant numbers: 81702216 and 81571828), the construction of famous traditional Chinese medicine doctor "Mo Wen" and workshop in Pudong New Area (Number: PDZYXK-3-2014018).

\section{Availability of data and materials}

All the data and materials can be found in the manuscript.

\section{Ethics approval and consent to participate}

The study was approved by the Ethics Committee of the Second Affiliated Hospital of Nantong University, LongHua Hospital, Shenzhen Luohu Hospital, and the First Affiliated Hospital of Zhengzhou University.

\section{Consent for publication}

All individual persons consented for their data to be published.

\section{Competing Interests}

The authors declare that they have no competing interests.

\section{References}

1. Prasad D, Schiff D. Malignant spinal-cord compression. Lancet Oncol. 2005;6(1):15-24.

2. Huisman $M$, van der Velden JM, van Vulpen $M$, van den Bosch MA, Chow E, Oner FC, et al. Spinal instability as defined by the spinal instability neoplastic score is associated with radiotherapy failure in metastatic spinal disease. Spine J. 2014;14(12):2835-40.

3. Margery J, Grassin F, Le Moulec S, Ruffie P. [Spinal cord compression from a malignant pleural mesothelioma]. Rev Pneumol Clin. 2005;61(2):112-4.

4. Fisher CG, DiPaola CP, Ryken TC, Bilsky MH, Shaffrey Cl, Berven SH, et al. A novel classification system for spinal instability in neoplastic disease: an evidence-based approach and expert consensus from the Spine Oncology Study Group. Spine (Phila Pa 1976). 2010;35(22):E1221-9.

5. Weber MH, Burch S, Buckley J, Schmidt MH, Fehlings MG, Vrionis FD, et al. Instability and impending instability of the thoracolumbar spine in patients with spinal metastases: a systematic review. Int J Oncol. 2011;38(1):5-12.

6. Fourney DR, Frangou EM, Ryken TC, Dipaola CP, Shaffrey Cl, Berven SH, et al. Spinal instability neoplastic score: an analysis of reliability and validity from the spine oncology study group. J Clin Oncol. 2011;29(22):3072-7.

7. Kearney R, Blondeau F, McPherson P, Bell A, Servant F, Drapeau M, et al. Elimination of redundant protein identifications in high throughput proteomics. Conf Proc IEEE Eng Med Biol Soc. 2005;5:4803-6. 
8. Schuck P. Assessing reproducibility for interval data in health-related quality of life questionnaires: which coefficient should be used? Qual Life Res. 2004;13(3):571-86.

9. Cronbach LJ, MeehI PE. Construct validity in psychological tests. Psychol Bull. 1955;52(4):281-302.

10. Landis JR, Koch GG. The measurement of observer agreement for categorical data. Biometrics. 1977;33(1):159-74.

11. Terwee CB, Bot SD, de Boer MR, van der Windt DA, Knol DL, Dekker J, et al. Quality criteria were proposed for measurement properties of health status questionnaires. $\mathrm{J}$ Clin Epidemiol. 2007;60(1):34-42.

12. Feise RJ, Michael Menke J. Functional rating index: a new valid and reliable instrument to measure the magnitude of clinical change in spinal conditions. Spine (Phila Pa 1976). 2001;26(1):78-86; discussion 7.

13. Fleiss JL, Shrout PE. The effects of measurement errors on some multivariate procedures. Am J Public Health. 1977;67(12):1188-91.

14. Xu C, Yin M, Sun Z, Yan Y, Mo W, Yan W. An Independent Interobserver Reliability and Intraobserver Reproducibility Evaluation of Spinal Instability Neoplastic Score and Kostuik Classification Systems for Spinal Tumor. World Neurosurg. 2020.

15. Pennington Z, Ahmed AK, Cottrill E, Westbroek EM, Goodwin ML, Sciubba DM. Intra- and interobserver reliability of the Spinal Instability Neoplastic Score system for instability in spine metastases: a systematic review and meta-analysis. Ann Transl Med. 2019;7(10):218.

16. Fox S, Spiess M, Hnenny L, Fourney DR. Spinal Instability Neoplastic Score (SINS): Reliability Among Spine Fellows and Resident Physicians in Orthopedic Surgery and Neurosurgery. Global Spine J. 2017;7(8):744-8.

17. Arana E, Kovacs FM, Royuela A, Asenjo B, Perez-Ramirez U, Zamora J. Spine Instability Neoplastic Score: agreement across different medical and surgical specialties. Spine J. 2016;16(5):591-9.

18. Yin M, Huang Q, Sun Z, Gao X, Chen G, He S, et al. An independent evaluation on the interobserver reliability and intraobserver reproducibility of Toyama classification system for cervical dumbbell tumors. Medicine (Baltimore). 2017;96(10):e6183. 